\title{
Estimating the Prevalence of Transthyretin Amyloid Cardiomyopathy in a Large In-Hospital Database in Japan
}

Ian Winburn (D) - Tomonori Ishii - Takuma Sumikawa •

Kanae Togo $\cdot$ Hideo Yasunaga

Received: June 7, 2019 / Published online: August 2, 2019

(C) The Author(s) 2019

\section{ABSTRACT}

Introduction: Transthyretin amyloid cardiomyopathy (ATTR-CM) - a debilitating, fatal disease resulting from the deposition of transthyretin (TTR) amyloid fibrils-can be hereditary due to mutations in the TTR gene (ATTRm) or wild type (ATTRwt). The global prevalence of ATTR-CM is largely unknown, although likely underestimated, with no formal epidemiological prevalence studies in Japan. This study aimed to estimate the prevalence of ATTR-CM in a large in-hospital database in Japan.

Methods: This was a retrospective, observational, cross-sectional study which utilized data from all adult patients (aged $\geq 20$ years) in the

Enhanced Digital Features To view enhanced digital features for this article go to https://doi.org/10.6084/ m9.figshare. 8869757.

Electronic Supplementary Material The online version of this article (https://doi.org/10.1007/s40119019-0142-5) contains supplementary material, which is available to authorized users.

I. Winburn $(\square)$

Pfizer, Walton Oaks, UK

e-mail: ian.winburn@pfizer.com

T. Ishii $\cdot$ T. Sumikawa $\cdot$ K. Togo

Pfizer Japan Inc., Tokyo, Japan

H. Yasunaga

Department of Clinical Epidemiology and Health

Economics, School of Public Health, University of

Tokyo, Tokyo, Japan hospital-based Japan Medical Data Vision (MDV) database from January 2010 to September 2018 to estimate the number of currently diagnosed ATTR-CM patients and describe their demographic and clinical characteristics and diagnostic modalities. ATTR-CM patients (ATTRwt and ATTRm) were identified using a range of diagnosis codes that were applied to create broad and narrow definitions of the disease.

Results: Over the 9 years of this study, there were 3255 (155.8 per million adult patients in the MDV database) to 3992 (191.1 per million) diagnoses of ATTRwt and 67 (3.2 per million) to 106 (5.1 per million) diagnoses of ATTRm in the MDV database (based on the narrow and broad definitions, respectively). There were 444 (21.2 per million) diagnoses of amyloid light-chain (AL) amyloidosis. Considering only those patients who were also diagnosed with heart failure, there were 1468 (70.3 per million) to 1798 (86.1 per million) diagnoses of ATTRwt and 50 (2.4 per million) to 61 (2.9 per million) diagnoses of ATTRm. Most ATTRwt patients ( 90\%) did not have a record of endomyocardial or abdominal wall biopsy, or of scintigram. Conclusion: This retrospective study provides an estimate of the number of patients diagnosed with ATTR-CM in a large in-hospital database in Japan over a period of 9 years. Improving awareness of disease prevalence may improve diagnosis and treatment.

Funding: Pfizer. 
Keywords: Amyloidosis; Cardiomyopathy; Japan; Observational study

\section{INTRODUCTION}

Transthyretin amyloid cardiomyopathy (ATTR$\mathrm{CM}$ ) is a debilitating, fatal disease resulting from the deposition of transthyretin amyloid fibrils, primarily in the heart [1]. ATTR-CM can be hereditary, due to mutations in the transthyretin (TTR) gene (ATTRm), or wild type (ATTRwt) $[2,3]$. The prevalence of ATTR-CM is largely unknown, with current estimates likely understating the actual prevalence as a consequence of phenotypic variability, nonspecific symptoms, a historical lack of an effective treatment, and limited awareness of the disease. For example, symptoms associated with ATTR-CM may also be observed in patients with heart failure with preserved ejection fraction or hypertensive heart disease, leading to misdiagnosis.

ATTR-CM is most common in older ( $>60$ years of age) males [1] and, despite having the highest proportion of older people in the world and a high prevalence of heart failure [5], there have not been any formal epidemiological studies investigating the prevalence of ATTR$\mathrm{CM}$ in Japan. Published estimates of the prevalence of ATTRwt in Japan are limited by the lack of consensus on the definition and characterization of this disease, and show a broad range. For example, 34 of 100 Japanese patients with idiopathic carpal tunnel syndrome were shown to have wild-type transthyretin deposition in one study [6], while a nationwide survey identified 51 ATTRwt patients across 2300 clinical departments in Japan [7]. Another study of 9508 patients who underwent endomyocardial biopsy between 2010 and 2013 in Japan found that 403 had a diagnosis of "amyloidosis," although not specifically ATTR-CM [8].

This study aimed to utilize the hospitalbased Japan Medical Data Vision (MDV) database to estimate the prevalence of patients diagnosed with ATTR-CM in a large in-hospital database in Japan over a period of 9 years.

\section{METHODS}

\section{Study Design}

This was a retrospective, observational, crosssectional study that utilized anonymized data from all adult patients in the Japan MDV database. The MDV database is a hospital-based database containing inpatient and outpatient hospital data, demographics, diagnoses, patient encounters and medical practices, medications, laboratory tests, and costs. As of September 2018, the database covers around 25 million accumulated patients of all ages (the total population of Japan is 126 million [9]) from over 370 hospitals distributed throughout Japan that are capable of treating advanced-stage patients, including (but not limited to) acute care facilities. All hospitals in the MDV database have $\geq 20$ beds. All patient data are anonymized with no personal data, so informed consent was not required. This article is based on previously conducted studies and does not contain any studies with human participants or animals performed by any of the authors.

The primary objective of this study was to estimate the prevalence of ATTR-CM in a large in-hospital database in Japan based on the number of diagnosed patients in the MDV database over a period of 9 years. Secondary objectives were to describe the demographic and clinical disposition of patients diagnosed with ATTR-CM, characterize the diagnostic modalities used for patients with ATTR-CM, and describe the prevalence of other subtypes of cardiac amyloidosis (specifically, amyloid lightchain [AL] amyloidosis).

\section{Data Analysis}

All patients with $\geq 1$ record in the MDV database between January 2010 and September 2018 who were $\geq 20$ years of age at the time of their ATTR-CM diagnosis or their first record in the MDV database were included in this analysis. ATTR-CM and comorbidities were identified using diagnosis codes (International Classification of Diseases [ICD], 10th revision) and disease names coded according to Japanese claims 
codes available in the MDV database. Cases of ATTR-CM were identified using both a broad definition (using a wider range of diagnosis codes which may only be suggestive of ATTR$\mathrm{CM}$ ) and a narrow definition. The two definitions were used in order to identify a range of prevalences, allowing us to make some allowance for misdiagnosis or misrecording (see Table S1 in the Electronic supplementary material, ESM, for a list of all diagnosis codes). Patients were first selected based on the presence of $\geq 1$ diagnosis code for ATTR-CM, and were grouped into those with any diagnosis code(s) under the broad definition and into a subset of those who only had diagnosis codes associated with the narrow definition (Fig. 1). These patients were then separated into the ATTRwt patients who did not have a diagnosis code for neuropathy and did not have a diagnosis code for AL amyloidosis or multiple myeloma, and the ATTRm patients who had a diagnosis code for neuropathy and did not have a diagnosis code for AL amyloidosis or multiple myeloma. Patients with a diagnosis of AL amyloidosis were also recorded. Demographics, including medical history and hospital data, were recorded for each patient. Prevalence was estimated for each year and over the whole period, and was expressed as the number of cases per 1 million adult patients in the MDV database (per million). Diagnosis codes and demographics for the subgroup of patients also diagnosed with heart failure were also assessed. The demographic and clinical characteristics of the cases are expressed as means and standard

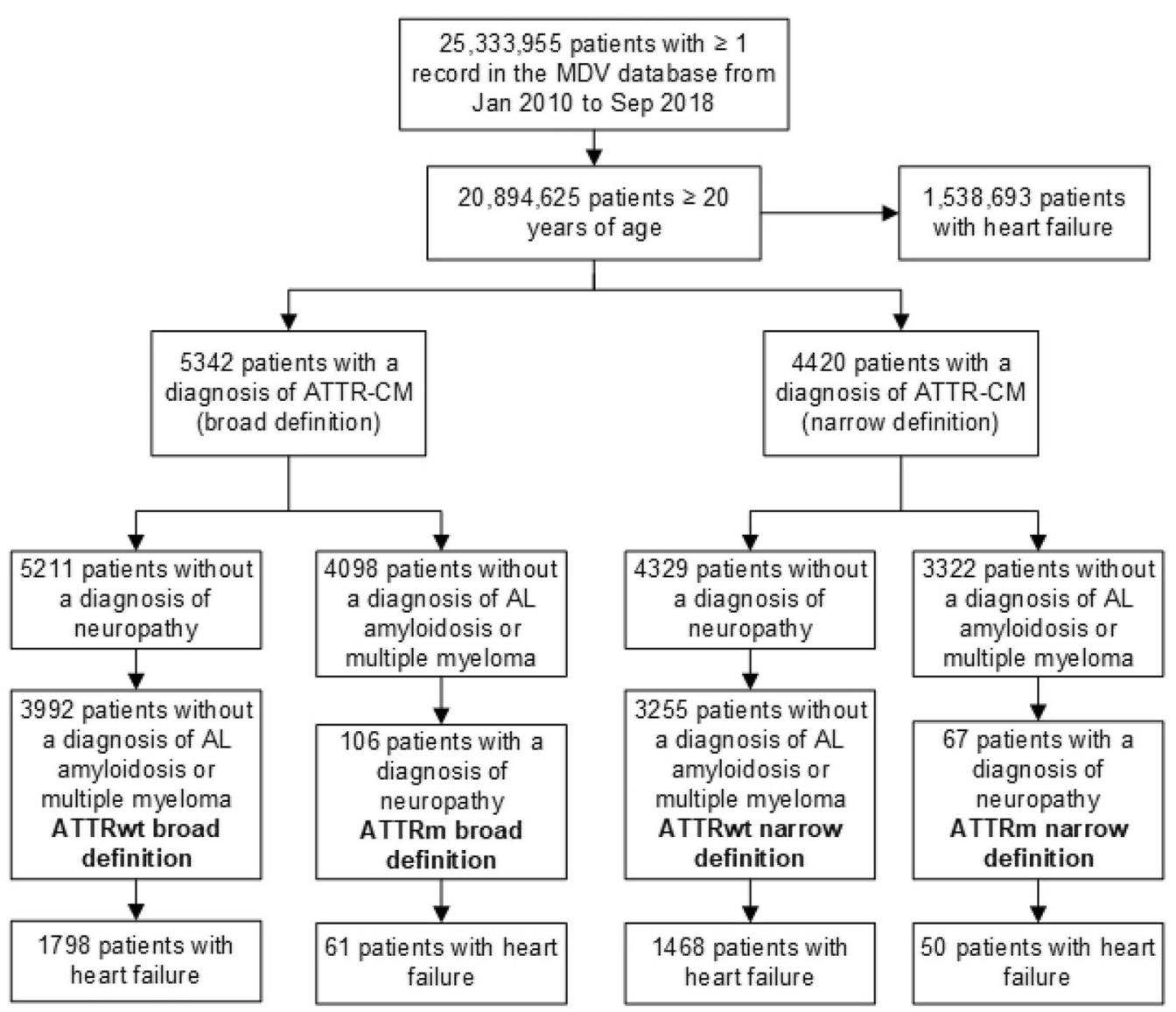

Fig. 1 Selection steps for patients with ATTRwt and ATTRm under the broad and narrow definitions. $A L$ amyloid light-chain, ATTR-CM transthyretin amyloid

cardiomyopathy, ATTRm hereditary transthyretin amyloidosis, ATTRwt wild-type transthyretin amyloid cardiomyopathy, $M D V$ Medical Data Vision 


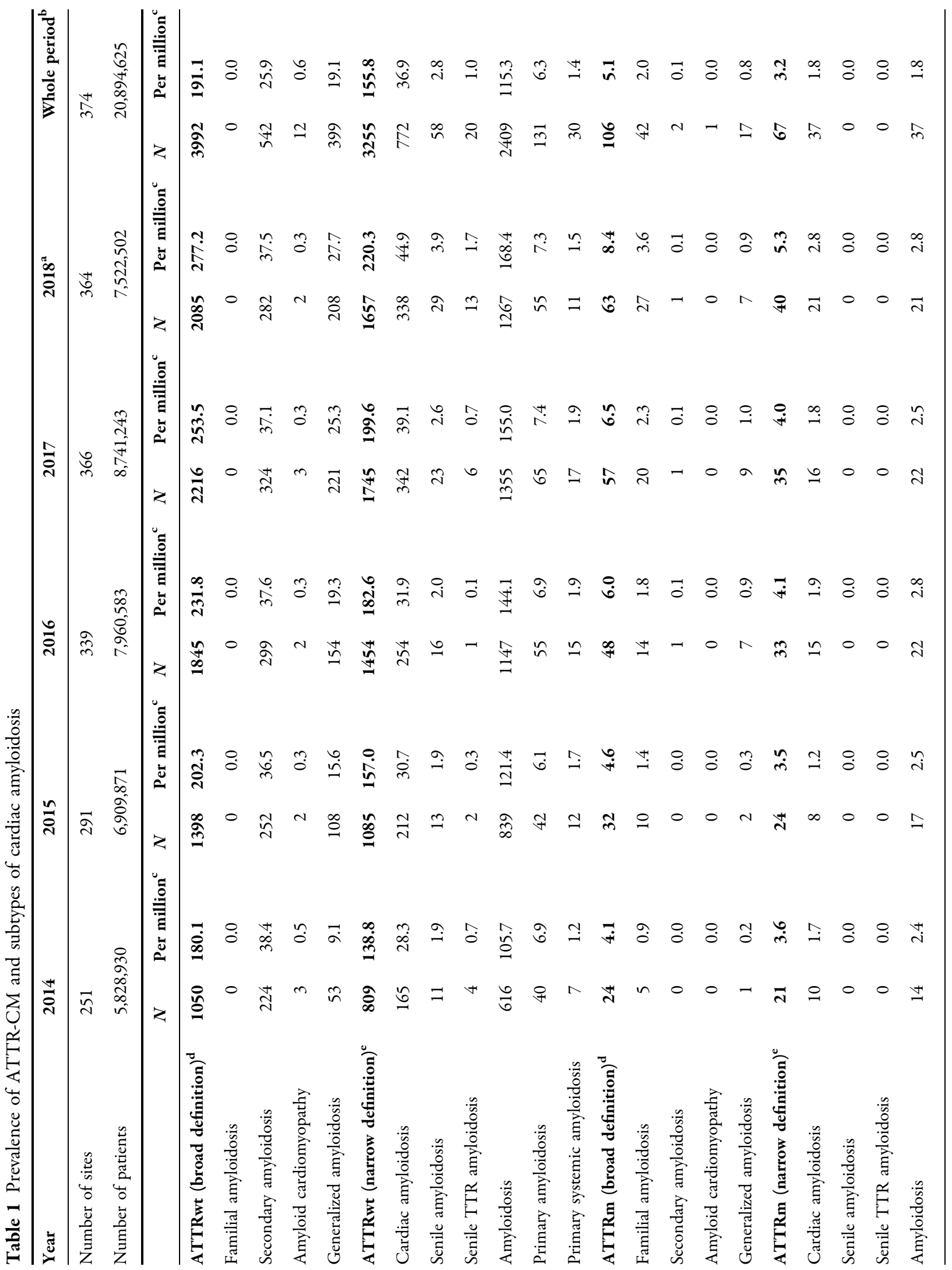




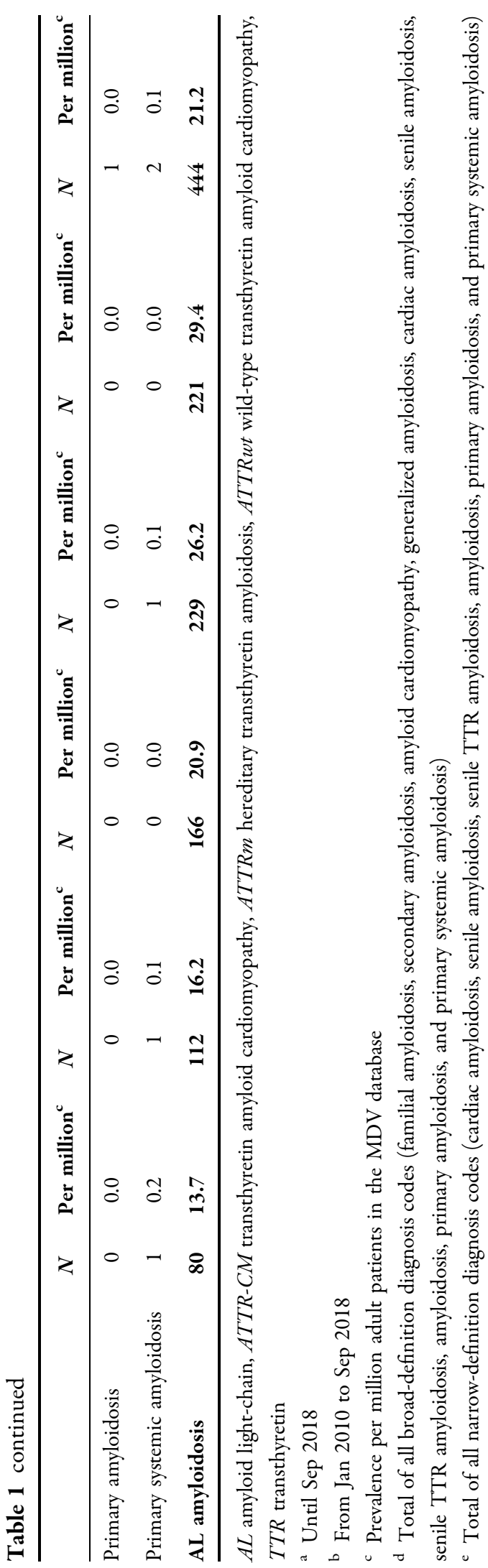

deviations (SDs) for continuous variables and as counts and percentages for categorical variables.

\section{RESULTS}

\section{Prevalence of ATTR-CM and Subtypes of Cardiac Amyloidosis}

The total number of patient records in the MDV database between January 2010 and September 2018 was $25,333,955$, of whom 20,894,625 were aged $\geq 20$ years (Fig. 1 ). Of these, the majority $(69.5 \%)$ were outpatients only, while $30.4 \%$ had $\geq 1$ inpatient visit and $4.4 \%$ had $\geq 1$ inpatient visit only (no outpatient visit). The mean (SD) age of all patients in the database was 57.7 (18.9) years and $46.3 \%$ were male. The majority of patients in the database $(59.3 \%)$ were from hospitals of an intermediate size (200-499 beds), while $29.6 \%$ were from hospitals with $\geq 500$ beds and $11.1 \%$ were from hospitals with $\leq 199$ beds.

Over the whole period (January 2010 to September 2018), there were 3992 diagnoses of ATTRwt and 106 diagnoses of ATTRm using the broad definition of ATTR-CM. Using the narrow definition of ATTR-CM, there were 3255 diagnoses of ATTRwt and 67 diagnoses of ATTRm (Fig. 1, Table 1). There were 444 diagnoses of AL amyloidosis.

The most common diagnosis code for patients with ATTRwt was "amyloidosis," representing over half of the diagnosed patients, followed by "cardiac amyloidosis" and "secondary amyloidosis." The most common diagnosis code for patients with ATTRm was "familial amyloidosis," followed by "cardiac amyloidosis" and "amyloidosis" (Table 1). The number of sites and number of patients in the MDV database increased each year. From 2013, the prevalence of ATTRwt and ATTRm as recorded in the database increased each year, as did the number of sites in the database (Fig. 2).

A total of 1,538,693 patients in the database had a diagnosis of heart failure (Fig. 1). Considering only patients who were also diagnosed with heart failure, there were 1798 (86.1 per million) diagnoses of ATTRwt and 61 (2.9 per million) diagnoses of ATTRm using the broad 

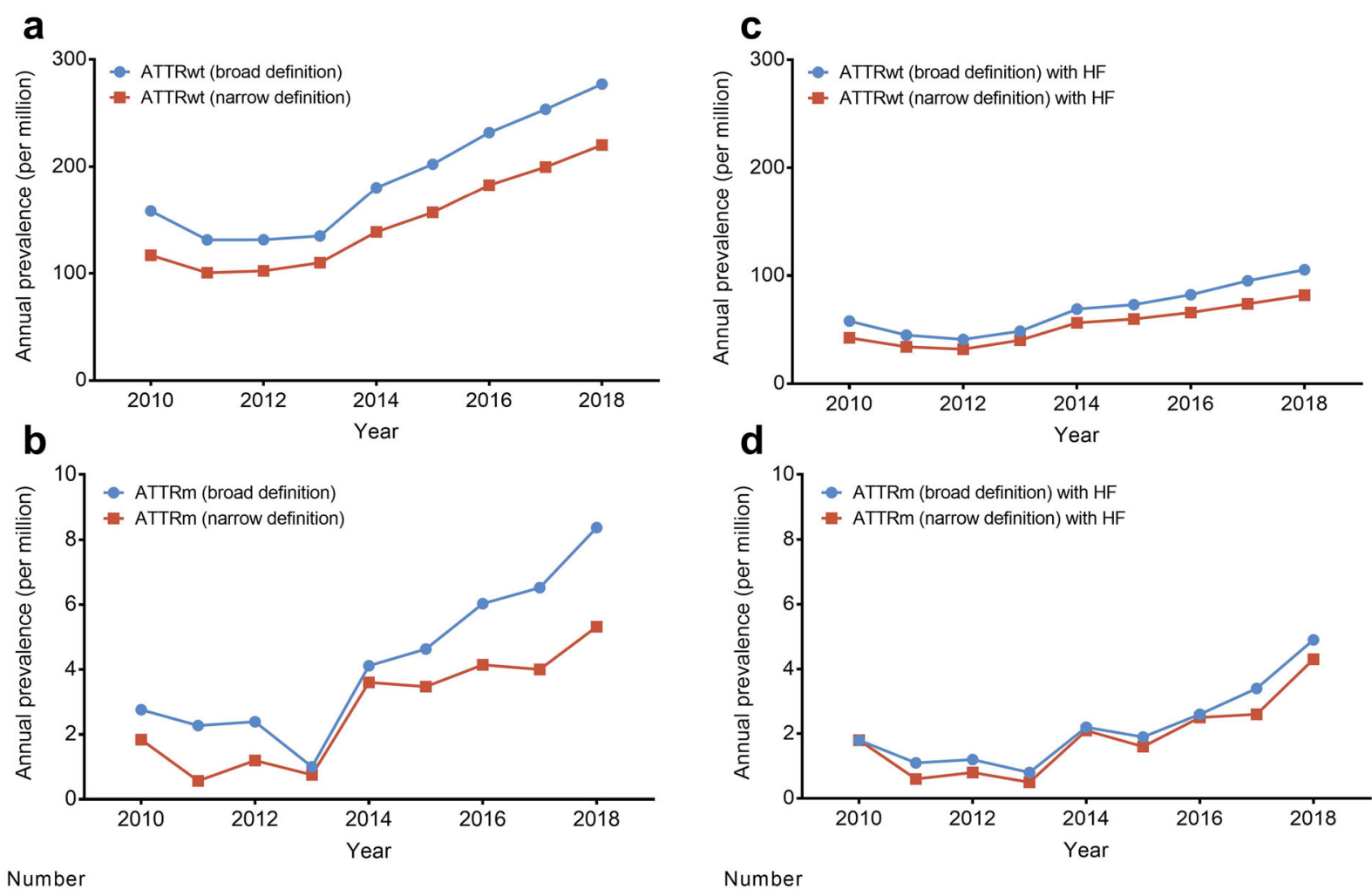

$\begin{array}{llllllllll}\text { of sites: } & 57 & 81 & 118 & 168 & 251 & 291 & 339 & 366 & 364\end{array}$

Fig. 2 Annual prevalences of a ATTRwt, b ATTRm, c ATTRwt with heart failure, and $\mathbf{d}$ ATTRm with heart failure per million adult patients in the MDV database. 2018 prevalence data covers the period from Jan 2010 to

definition of ATTR-CM. Using the narrow definition of ATTR-CM, there were 1468 (70.3 per million) diagnoses of ATTRwt and 50 (2.4 per million) diagnoses of ATTRm (Table 2). There were 308 (14.7 per million) diagnoses of $\mathrm{AL}$ amyloidosis.

\section{Demographic Characteristics of Patients with Cardiac Amyloidosis}

Under the broad definition, and over the whole period, greater than half $(53.7 \%)$ of the ATTRwt patients were female (Table 3), while the majority of both the ATTRm patients $(61.3 \%$; Table 4) and the AL amyloidosis patients (59.2\%; Table 5) were male. ATTRm patients tended to be younger (mean [SD] age, 63.7 [15.2] years) than ATTRwt (68.1 [14.2] years) and AL amyloidosis (68.8 [11.1]) patients. The

Number

$\begin{array}{llllllllll}\text { of sites: } & 57 & 81 & 118 & 168 & 251 & 291 & 339 & 366 & 364\end{array}$

Sep 2018. ATTRm hereditary transthyretin amyloidosis, ATTRwt wild-type transthyretin amyloid cardiomyopathy, $H F$ heart failure

proportion of the patients aged $\geq 80$ years was higher for the ATTRwt patients (23.0\%) than for the ATTRm $(9.4 \%)$ or AL amyloidosis (16.9\%) patients. The proportion of patients with ATTRwt tended to increase with age $(76.9 \%$ of the ATTRwt patients were aged $\geq 60$ years), and this trend was observed for every ATTR-CM diagnosis code.

$\mathrm{AL}$ amyloidosis patients were more likely to have $\geq 1$ hospital visit (84.2\%) than ATTRwt $(62.6 \%)$ or ATTRm (63.2\%) patients. AL amyloidosis patients were also more likely to have their claims data from a larger hospital than ATTRm patients who were, in turn, more likely have their claims data from a larger hospital than ATTRwt patients. However, each group was more likely to be from a larger hospital than the 20,894,625 patients in the whole MDV database, $11.1 \%$ of whom were from a hospital with $\leq 199$ beds, $59.3 \%$ were from a 200 - to 


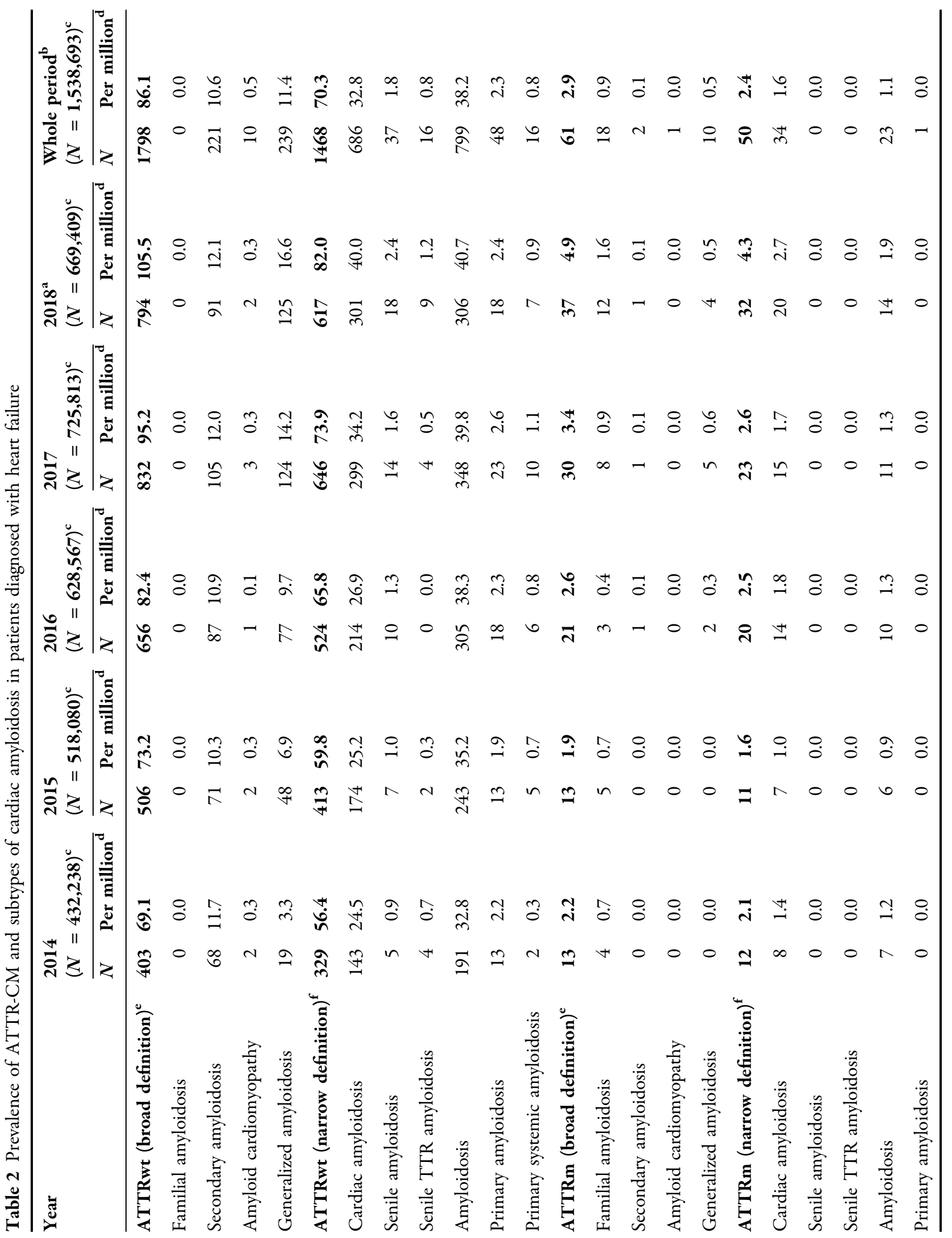




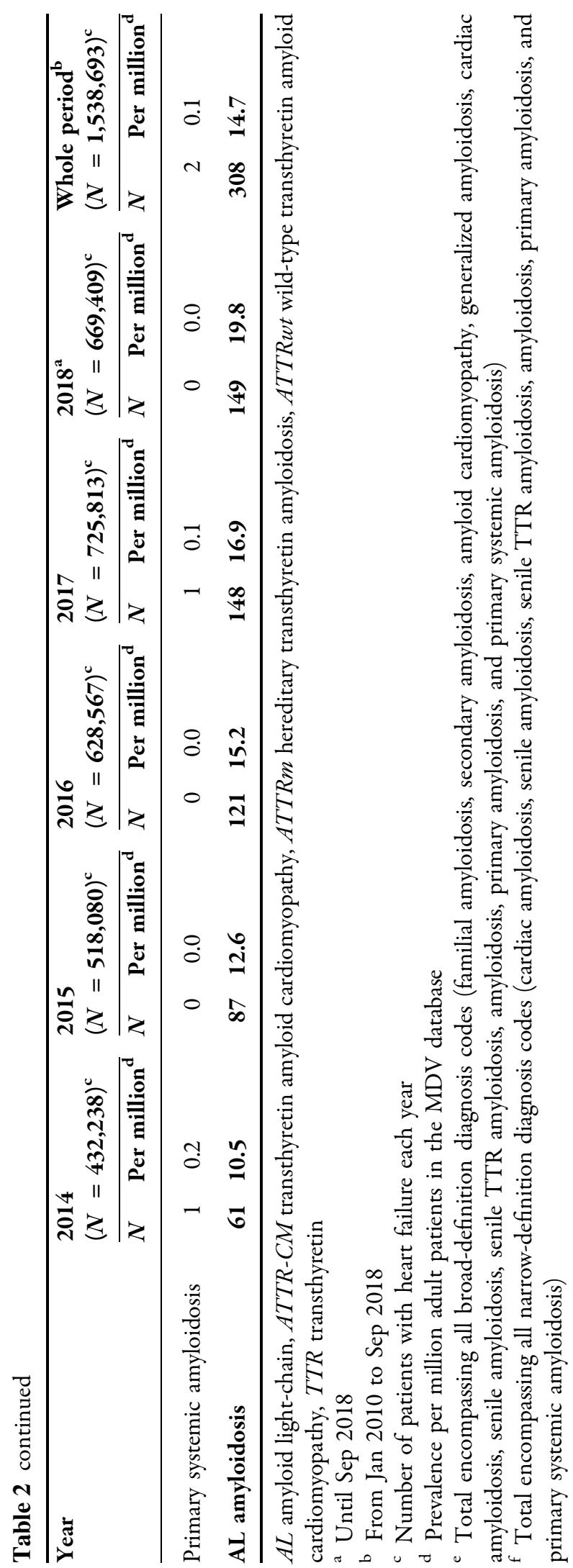

499-bed hospital, and $29.6 \%$ were from a $\geq 500$ bed hospital. Compared with patients in the whole MDV database, each group was also more likely to have died in the hospital $(2.7 \%, 12.8 \%$, $8.5 \%$, and $22.5 \%$ for the whole MDV database, ATTRwt, ATTRm, and AL amyloidosis, respectively) and more likely to be from a university hospital $(5.9 \%, 12.8 \%, 16.0 \%$, and $9.7 \%$ for the whole MDV database, ATTRwt, ATTRm, and AL amyloidosis, respectively).

Considering only those patients with a diagnosis of heart failure, demographic characteristics were largely similar to those of all ATTR-CM patients (Table 6). There remained a relatively high proportion of female ATTRwt patients $(44.8 \%$, compared with $53.7 \%$ of all ATTRwt patients). However, ATTRwt patients with heart failure were more likely to have had $\geq 1$ inpatient visit $(82.3 \%$ compared with $62.6 \%)$ or to have died in hospital $(21.1 \%$ compared with 12.8\%).

\section{Diagnostic Modalities in Patients with ATTR-CM (Broad Definition)}

The large majority of patients diagnosed with ATTR-CM did not have a record of a tissue biopsy or a scintigram with sodium pyrophosphate. The greater proportion of those who did were inpatients (Table 7). Approximately 9\% of all ATTRwt patients (both inpatients and outpatients) had an endomyocardial biopsy or abdominal wall biopsy at any time. Digestive tract biopsy was more common (1011 patients, $25.3 \%$ ), but it was rare for patients with a digestive tract biopsy to also have an endomyocardial biopsy (59 patients) or abdominal wall biopsy (65 patients), which would be required to optimally confirm a diagnosis of ATTRwt.

Diagnostic modalities were largely similar in the subgroup of patients diagnosed with heart failure, with digestive tract biopsy and scintigram with sodium pyrophosphate slightly more common (Table 8).

Considering only diagnostic modalities within 8 weeks of diagnosis in all patients, 121 (3.0\%) ATTRwt patients had an endomyocardial biopsy, 41 (1.0\%) had an abdominal wall biopsy, and $407(10.2 \%)$ had a digestive tract 
Table 3 Demographic characteristics of the ATTRwt patients (broad definition)

\begin{tabular}{|c|c|c|c|c|c|c|}
\hline & $\begin{array}{l}2014 \\
(N=1050)\end{array}$ & $\begin{array}{l}2015 \\
(N=1398)\end{array}$ & $\begin{array}{l}2016 \\
(N=1845)\end{array}$ & $\begin{array}{l}2017 \\
(N=2216)\end{array}$ & $\begin{array}{l}2018^{a} \\
(N=2085)\end{array}$ & $\begin{array}{l}\text { Whole period }^{\mathbf{b}} \\
(N=3992)\end{array}$ \\
\hline Age (years), mean (SD) & $67.19(14.1)$ & $67.73(13.5)$ & $67.51(13.8)$ & $67.53(14.1)$ & $68.26(13.9)$ & $68.07(14.2)$ \\
\hline Male, $n(\%)$ & $460(43.8)$ & $586(41.9)$ & $773(41.9)$ & $948(42.8)$ & $902(43.3)$ & $1849(46.3)$ \\
\hline Female, $n(\%)$ & $590(56.2)$ & $812(58.1)$ & $1072(58.1)$ & $1268(57.2)$ & $1183(56.7)$ & $2143(53.7)$ \\
\hline \multicolumn{7}{|l|}{ Comorbidities $^{c}, n(\%)$} \\
\hline Heart failure & $403(38.4)$ & $506(36.2)$ & $656(35.6)$ & $832(37.6)$ & $794(38.1)$ & $1798(45.0)$ \\
\hline Arrhythmia & $119(11.3)$ & $143(10.2)$ & $225(12.2)$ & $286(12.9)$ & $253(12.1)$ & $602(15.1)$ \\
\hline Atrial fibrillation or flutter & $143(13.6)$ & $193(13.8)$ & $236(12.8)$ & $294(13.3)$ & $321(15.4)$ & $686(17.2)$ \\
\hline Hypertension & $610(58.1)$ & $770(55.1)$ & $943(51.1)$ & $1147(51.8)$ & $1103(52.9)$ & $2204(55.2)$ \\
\hline Chronic kidney disease III-V & $321(30.6)$ & $367(26.3)$ & $450(24.4)$ & $514(23.2)$ & $457(21.9)$ & $1079(27.0)$ \\
\hline Angina & $237(22.6)$ & $296(21.2)$ & $358(19.4)$ & $437(19.7)$ & $406(19.5)$ & $935(23.4)$ \\
\hline Diabetes mellitus & $420(40.0)$ & $530(37.9)$ & $718(38.9)$ & $892(40.3)$ & $873(41.9)$ & $1624(40.7)$ \\
\hline Malignancy & $175(16.7)$ & $211(15.1)$ & $315(17.1)$ & $380(17.2)$ & $334(16.0)$ & $767(19.2)$ \\
\hline \multicolumn{7}{|l|}{ Procedures, $n(\%)$} \\
\hline Carpal tunnel release & $6(0.6)$ & $4(0.3)$ & $3(0.2)$ & $11(0.5)$ & $13(0.6)$ & $58(1.5)$ \\
\hline Surgical aortic valve replacement & $1(0.1)$ & $0(0.0)$ & $0(0.0)$ & $13(0.6)$ & $1(0.1)$ & $17(0.4)$ \\
\hline $\begin{array}{l}\text { Transcatheter aortic valve } \\
\text { implantation }\end{array}$ & $0(0.0)$ & $0(0.0)$ & $0(0.0)$ & $13(0.6)$ & $0(0.0)$ & $15(0.4)$ \\
\hline \multicolumn{7}{|l|}{ Heart failure medication use, $n(\%)$} \\
\hline Beta-blocker & $186(17.7)$ & $234(16.7)$ & $279(15.1)$ & $362(16.3)$ & $347(16.6)$ & $936(23.5)$ \\
\hline ACE inhibitors & $63(6.0)$ & $89(6.4)$ & $103(5.6)$ & $140(6.3)$ & $121(5.8)$ & $394(9.9)$ \\
\hline $\mathrm{ARBs}$ & $275(26.2)$ & $343(24.5)$ & $386(20.9)$ & $462(20.9)$ & $398(19.1)$ & $1052(26.4)$ \\
\hline Calcium channel blocker & $297(28.3)$ & $376(26.9)$ & $447(24.2)$ & $542(24.5)$ & $481(23.1)$ & $1360(34.1)$ \\
\hline Diuretic & $281(26.8)$ & $351(25.1)$ & $410(22.2)$ & $532(24.0)$ & $493(23.7)$ & $1414(35.4)$ \\
\hline Aldosterone antagonists & $103(9.8)$ & $141(10.1)$ & $175(9.5)$ & $259(11.7)$ & $218(10.5)$ & $688(17.2)$ \\
\hline$\geq 1$ inpatient visit, $n(\%)$ & $440(41.9)$ & $538(38.5)$ & $621(33.7)$ & $780(35.2)$ & $597(28.6)$ & $2497(62.6)$ \\
\hline Death at discharge, $n(\%)$ & $58(5.5)$ & $71(5.1)$ & $80(4.3)$ & $99(4.5)$ & $64(3.1)$ & $512(12.8)$ \\
\hline \multicolumn{7}{|l|}{ Hospital size, $n(\%)$} \\
\hline$\leq 199$ beds & $99(9.4)$ & $117(8.4)$ & $119(6.5)$ & $132(6.0)$ & $119(5.7)$ & $296(7.4)$ \\
\hline $200-499$ beds & $484(46.1)$ & $740(52.9)$ & $900(48.8)$ & $1070(48.3)$ & $1084(52.0)$ & $2055(51.5)$ \\
\hline$\geq 500$ beds & $467(44.5)$ & $541(38.7)$ & $826(44.8)$ & $1014(45.8)$ & $882(42.3)$ & $1641(41.1)$ \\
\hline \multicolumn{7}{|l|}{ Hospital administration, $n(\%)$} \\
\hline University & $184(17.5)$ & $228(16.3)$ & $252(13.7)$ & $313(14.1)$ & $262(12.6)$ & $511(12.8)$ \\
\hline Public (local) & $242(23.0)$ & $300(21.5)$ & $527(28.6)$ & $657(29.7)$ & $623(29.9)$ & $1064(26.7)$ \\
\hline
\end{tabular}


Table 3 continued

\begin{tabular}{|c|c|c|c|c|c|c|}
\hline & $\begin{array}{l}2014 \\
(N=1050)\end{array}$ & $\begin{array}{l}2015 \\
(N=1398)\end{array}$ & $\begin{array}{l}2016 \\
(N=1845)\end{array}$ & $\begin{array}{l}2017 \\
(N=2216)\end{array}$ & $\begin{array}{l}2018^{\mathrm{a}} \\
(N=2085)\end{array}$ & $\begin{array}{l}\text { Whole period }{ }^{\mathrm{b}} \\
(N=3992)\end{array}$ \\
\hline Private & $193(18.4)$ & $220(15.7)$ & $304(16.5)$ & $352(15.9)$ & $325(15.6)$ & $749(18.8)$ \\
\hline Public (government) & $431(41.1)$ & $650(46.5)$ & $762(41.3)$ & $894(40.3)$ & $875(42.0)$ & $1668(41.8)$ \\
\hline
\end{tabular}

$A C E$ angiotensin-converting enzyme, $A R B$ angiotensin receptor blocker, $A T T R w t$ wild-type transthyretin amyloid cardiomyopathy, $S D$ standard deviation

a Until Sep 2018

b From Jan 2010 to Sep 2018

c Comorbidities occurring in $>15 \%$ of patients over the whole period

biopsy, while $14(0.4 \%)$ had a scintigram. Among the patients with a digestive tract biopsy, endomyocardial biopsy (21 of 407 patients) and abdominal wall biopsy (17 of 407 patients) were rare.

\section{DISCUSSION}

This retrospective, observational, cross-sectional study utilized a large database of over 25 million patients from over 370 Japanese hospitals to estimate the prevalence of patients diagnosed with ATTR-CM in a large in-hospital database in Japan. The use of broad and narrow definitions for ATTR-CM led to a range of estimates of the number of ATTRwt patients in the MDV database over the 9 years of this analysis of between 3255 (155.8 per million) and 3992 (191.1 per million). ATTRm was less common, with between 67 (3.2 per 1 million) and 106 (5.1 per million) patients in the MDV database. Among patients who were also diagnosed with heart failure, who were potentially more likely to represent symptomatic ATTR-CM, there were between 1468 (70.3 per million) and 1798 (86.1 per million) diagnoses of ATTRwt, and between 50 (2.4 per million) and 61 (2.9 per million) diagnoses of ATTRm. The prevalence of ATTR$\mathrm{CM}$ in the MDV database was shown to increase over the duration of this study at the same time that the size of the database itself increased. This increase may most likely be related to an increase in awareness of the disease, including the recruitment processes for clinical trials in Japan that test new treatments for ATTR-CM.

Prior estimates of the prevalence of ATTRwt in Japan have identified as few as 51 total patients in over 2300 clinical departments in Japan [7]. This prior estimate was based on a questionnaire sent to clinical departments. The patients were identified in a limited number of institutes (ten in total), which led the authors to conclude that ATTRwt was underdiagnosed in Japan [7]. This study provides a significantly larger estimate over a 9-year period, but this is based on codes in a claims database which were not validated against the actual patient diagnosis, limiting the interpretation of these results. This limitation, together with the fact that many patients are likely undiagnosed and that the MDV database does not cover the entire population of Japan, prevents the calculation of the true prevalence of ATTR-CM in Japan and limits this analysis to an estimate.

Studies in other countries have reported the incidence of ATTRwt to be $16 \%$ in patients with severe aortic stenosis who were undergoing transcatheter aortic valve replacement [4], and $5 \%$ in patients with presumed hypertrophic cardiomyopathy [10]. In terms of individual patients identified, the Transthyretin Amyloidosis Outcomes Survey (THAOS) registered approximately 189 patients with ATTRwt in the United States [11] and 125 in continental Western Europe [12], while the United Kingdom National Amyloidosis Center enrolled 711 patients with ATTRwt between 2000 and 2017 [13]. While there are significant differences 
Table 4 Demographic characteristics of ATTRm patients (broad definition)

\begin{tabular}{|c|c|c|c|c|c|c|}
\hline & $\begin{array}{l}2014 \\
(N=24)\end{array}$ & $\begin{array}{l}2015 \\
(N=32)\end{array}$ & $\begin{array}{l}2016 \\
(N=48)\end{array}$ & $\begin{array}{l}2017 \\
(N=57)\end{array}$ & $\begin{array}{l}2018^{\mathrm{a}} \\
(N=63)\end{array}$ & $\begin{array}{l}\text { Whole period }^{\mathrm{b}} \\
(N=106)\end{array}$ \\
\hline Age (years), mean (SD) & $62.8(14.4)$ & $60.1(17.1)$ & $60.2(15.1)$ & $61.1(14.3)$ & $63.3(14.5)$ & $63.7(15.2)$ \\
\hline Male, $n(\%)$ & $9(37.5)$ & $15(46.9)$ & $28(58.3)$ & $35(61.4)$ & $44(69.8)$ & $65(61.3)$ \\
\hline Female, $n(\%)$ & $15(62.5)$ & $17(53.1)$ & $20(41.7)$ & $22(38.6)$ & $19(30.2)$ & $41(38.7)$ \\
\hline \multicolumn{7}{|l|}{ Comorbidities $^{\mathrm{c}}, n(\%)$} \\
\hline Heart failure & $13(54.2)$ & $13(40.6)$ & $21(43.8)$ & $30(52.6)$ & $37(58.7)$ & $61(57.6)$ \\
\hline Arrhythmia & $5(20.8)$ & $4(12.5)$ & $6(12.5)$ & $7(12.3)$ & $12(19.1)$ & $17(16.0)$ \\
\hline Hypertension & $8(33.3)$ & $10(31.3)$ & $17(35.4)$ & $22(38.6)$ & $25(39.7)$ & $48(45.3)$ \\
\hline Angina & $7(29.2)$ & $3(9.4)$ & $6(12.5)$ & $7(12.3)$ & $8(12.7)$ & $17(16.0)$ \\
\hline Diabetes mellitus & $3(12.5)$ & $7(21.9)$ & $10(20.8)$ & $13(22.8)$ & $15(23.8)$ & $31(29.3)$ \\
\hline \multicolumn{7}{|l|}{ Procedures, $n(\%)$} \\
\hline Carpal tunnel release & $0(0.0)$ & $0(0.0)$ & $0(0.0)$ & $0(0.0)$ & $0(0.0)$ & $1(0.9)$ \\
\hline Surgical aortic valve replacement & $0(0.0)$ & $0(0.0)$ & $0(0.0)$ & $0(0.0)$ & $0(0.0)$ & $0(0.0)$ \\
\hline $\begin{array}{l}\text { Transcatheter aortic valve } \\
\text { implantation }\end{array}$ & $0(0.0)$ & $0(0.0)$ & $0(0.0)$ & $0(0.0)$ & $0(0.0)$ & $0(0.0)$ \\
\hline \multicolumn{7}{|l|}{ Heart failure medication use, $n(\%)$} \\
\hline Beta-blocker & $2(8.3)$ & $3(9.4)$ & $5(10.4)$ & $6(10.5)$ & $8(12.7)$ & $21(19.8)$ \\
\hline ACE inhibitors & $2(8.3)$ & $0(0.0)$ & $4(8.3)$ & $1(1.8)$ & $4(6.4)$ & $11(10.4)$ \\
\hline $\mathrm{ARBs}$ & $2(8.3)$ & $4(12.5)$ & $2(4.2)$ & $3(5.3)$ & $6(9.5)$ & $16(15.1)$ \\
\hline Calcium channel blocker & $1(4.2)$ & $3(9.4)$ & $4(8.3)$ & $6(10.5)$ & $2(3.2)$ & $14(13.2)$ \\
\hline Diuretic & $5(20.8)$ & $8(25.0)$ & $12(25.0)$ & $14(24.6)$ & $20(31.8)$ & $44(41.5)$ \\
\hline Aldosterone antagonists & $3(12.5)$ & $2(6.3)$ & $4(8.3)$ & $4(7.0)$ & $7(11.1)$ & $21(19.8)$ \\
\hline$\geq 1$ inpatient visit, $n(\%)$ & $8(33.3)$ & $7(21.9)$ & $21(43.8)$ & $15(26.3)$ & $17(27.0)$ & $67(63.2)$ \\
\hline Death at discharge, $n(\%)$ & $1(4.2)$ & $1(3.1)$ & $2(4.2)$ & $1(1.8)$ & $2(3.2)$ & $9(8.5)$ \\
\hline \multicolumn{7}{|l|}{ Hospital size, $n(\%)$} \\
\hline$\leq 199$ beds & $1(4.2)$ & $1(3.1)$ & $3(6.3)$ & $1(1.8)$ & $2(3.2)$ & $5(4.7)$ \\
\hline $200-499$ beds & $10(41.7)$ & $17(53.1)$ & $23(47.9)$ & $28(49.1)$ & $29(46.0)$ & $48(45.3)$ \\
\hline$\geq 500$ beds & $13(54.2)$ & $14(43.8)$ & $22(45.8)$ & $28(49.1)$ & $32(50.8)$ & $53(50.0)$ \\
\hline \multicolumn{7}{|l|}{ Hospital administration, $n$ (\%) } \\
\hline University & $5(20.8)$ & $2(6.3)$ & $6(12.5)$ & $7(12.3)$ & $12(19.1)$ & $17(16.0)$ \\
\hline Public (local) & $11(45.8)$ & $18(56.3)$ & $25(52.1)$ & $22(38.6)$ & $32(50.8)$ & $41(38.7)$ \\
\hline Private & $0(0.0)$ & $2(6.3)$ & $3(6.3)$ & $7(12.3)$ & $5(7.9)$ & $11(10.4)$ \\
\hline Public (government) & $8(33.3)$ & $10(31.3)$ & $14(29.2)$ & $21(36.8)$ & $14(22.2)$ & $37(34.9)$ \\
\hline
\end{tabular}

$A C E$ angiotensin-converting enzyme, $A R B$ angiotensin receptor blocker, $A T T R m$ hereditary transthyretin amyloidosis, $S D$ standard deviation

a Until Sep 2018

b From Jan 2010 to Sep 2018

c Comorbidities occurring in $>15 \%$ of patients over the whole period 
Table 5 Demographic characteristics of AL amyloidosis patients

\begin{tabular}{|c|c|c|c|c|c|c|}
\hline & $\begin{array}{l}2014 \\
(N=80)\end{array}$ & $\begin{array}{l}2015 \\
(N=112)\end{array}$ & $\begin{array}{l}2016 \\
(N=166)\end{array}$ & $\begin{array}{l}2017 \\
(N=229)\end{array}$ & $\begin{array}{l}2018^{\mathrm{a}} \\
(N=221)\end{array}$ & $\begin{array}{l}\text { Whole period } \\
(N=444)\end{array}$ \\
\hline Age (years), mean (SD) & $68.5(9.2)$ & $68.0(11.5)$ & $68.3(11.3)$ & $67.9(10.9)$ & $68.5(11.1)$ & $68.8(11.1)$ \\
\hline Male, $n(\%)$ & $43(53.8)$ & $58(51.8)$ & $93(56.0)$ & $137(59.8)$ & $137(62.0)$ & $263(59.2)$ \\
\hline Female, $n(\%)$ & $37(46.3)$ & $54(48.2)$ & $73(44.0)$ & $92(40.2)$ & $84(38.0)$ & $181(40.8)$ \\
\hline \multicolumn{7}{|l|}{ Comorbidities $^{\mathrm{c}}, n(\%)$} \\
\hline Heart failure & $61(76.3)$ & $87(77.7)$ & $121(72.9)$ & $148(64.6)$ & $149(67.4)$ & $308(69.4)$ \\
\hline Arrhythmia & $17(21.3)$ & $23(20.5)$ & $32(19.3)$ & $28(12.2)$ & $34(15.4)$ & $69(15.5)$ \\
\hline Hypertension & $54(67.5)$ & $71(63.4)$ & $105(63.3)$ & $145(63.3)$ & $136(61.5)$ & $274(61.7)$ \\
\hline Chronic kidney disease III-V & $27(33.8)$ & $38(33.9)$ & $56(33.7)$ & $77(33.6)$ & $65(29.4)$ & $159(35.8)$ \\
\hline Angina & $31(38.8)$ & $36(32.1)$ & $55(33.1)$ & $60(26.2)$ & $50(22.6)$ & $128(28.8)$ \\
\hline Multiple myeloma & $59(73.8)$ & $85(75.9)$ & $132(79.5)$ & $172(75.1)$ & $163(73.8)$ & $317(71.4)$ \\
\hline Diabetes mellitus & $34(42.5)$ & $44(39.3)$ & $69(41.6)$ & $91(39.7)$ & $94(42.5)$ & $190(42.8)$ \\
\hline Malignancy & $66(82.5)$ & $92(82.1)$ & $141(84.9)$ & $186(81.2)$ & $175(79.2)$ & $344(77.5)$ \\
\hline \multicolumn{7}{|l|}{ Procedures, $n(\%)$} \\
\hline Carpal tunnel release & $0(0.0)$ & $0(0.0)$ & $1(0.6)$ & $1(0.4)$ & $0(0.0)$ & $5(1.1)$ \\
\hline Surgical aortic valve replacement & $0(0.0)$ & $0(0.0)$ & $0(0.0)$ & $0(0.0)$ & $0(0.0)$ & $0(0.0)$ \\
\hline $\begin{array}{l}\text { Transcatheter aortic valve } \\
\text { implantation }\end{array}$ & $0(0.0)$ & $0(0.0)$ & $0(0.0)$ & $0(0.0)$ & $0(0.0)$ & $0(0.0)$ \\
\hline \multicolumn{7}{|l|}{ Heart failure medication use, $n(\%)$} \\
\hline Beta-blocker & $21(26.3)$ & $36(32.1)$ & $42(25.3)$ & $43(18.8)$ & $38(17.2)$ & $115(25.9)$ \\
\hline ACE inhibitors & $6(7.5)$ & $6(5.4)$ & $8(4.8)$ & $14(6.1)$ & $22(10.0)$ & $50(11.3)$ \\
\hline ARBs & $18(22.5)$ & $31(27.7)$ & $39(23.5)$ & $50(21.8)$ & $35(15.8)$ & $132(29.7)$ \\
\hline Calcium channel blocker & $21(26.3)$ & $27(24.1)$ & $36(21.7)$ & $54(23.6)$ & $38(17.2)$ & $133(30.0)$ \\
\hline Diuretic & $46(57.5)$ & $76(67.9)$ & $96(57.8)$ & $111(48.5)$ & $105(47.5)$ & $294(66.2)$ \\
\hline Aldosterone antagonists & $25(31.3)$ & $31(27.7)$ & $39(23.5)$ & $47(20.5)$ & $37(16.7)$ & $140(31.5)$ \\
\hline$\geq 1$ inpatient visit, $n(\%)$ & $60(75.0)$ & $85(75.9)$ & $118(71.1)$ & $134(58.5)$ & $104(47.1)$ & $374(84.2)$ \\
\hline Death at discharge, $n(\%)$ & $5(6.3)$ & $16(14.3)$ & $25(15.1)$ & $21(9.2)$ & $9(4.1)$ & $100(22.5)$ \\
\hline \multicolumn{7}{|l|}{ Hospital size, $n(\%)$} \\
\hline$\leq 199$ beds & $1(1.3)$ & $1(0.9)$ & $5(3.0)$ & $6(2.6)$ & $8(3.6)$ & $13(2.9)$ \\
\hline 200-499 beds & $23(28.8)$ & $30(26.8)$ & $45(27.1)$ & $87(38.0)$ & $71(32.1)$ & $166(37.4)$ \\
\hline$\geq 500$ beds & $56(70.0)$ & $81(72.3)$ & $116(69.9)$ & $136(59.4)$ & $142(64.3)$ & $265(59.7)$ \\
\hline
\end{tabular}


Table 5 continued

\begin{tabular}{|c|c|c|c|c|c|c|}
\hline & $\begin{array}{l}2014 \\
(N=80)\end{array}$ & $\begin{array}{l}2015 \\
(N=112)\end{array}$ & $\begin{array}{l}2016 \\
(N=166)\end{array}$ & $\begin{array}{l}2017 \\
(N=229)\end{array}$ & $\begin{array}{l}2018^{\mathrm{a}} \\
(N=221)\end{array}$ & $\begin{array}{l}\text { Whole period } \\
(N=444)\end{array}$ \\
\hline \multicolumn{7}{|c|}{ Hospital administration, $n(\%)$} \\
\hline University & $7(8.8)$ & $17(15.2)$ & $14(8.4)$ & $19(8.3)$ & $21(9.5)$ & $43(9.7)$ \\
\hline Public (local) & $22(27.5)$ & $35(31.3)$ & $46(27.7)$ & $74(32.3)$ & $72(32.6)$ & $130(29.3)$ \\
\hline Private & $3(3.8)$ & $7(6.3)$ & $39(23.5)$ & $47(20.5)$ & $44(19.9)$ & $85(19.1)$ \\
\hline Public (government) & $48(60.0)$ & $53(47.3)$ & $67(40.4)$ & $89(38.9)$ & $84(38.0)$ & $186(41.9)$ \\
\hline
\end{tabular}

between a large observational study and the enrollment of patients in a prospective registry, it is possible that the higher prevalence of ATTR-CM in Japan identified in this study is an overestimate.

There were notable demographic and clinical differences between the patients with ATTRwt identified in this study and past descriptions of this population. For example, only $1.5 \%$ of the ATTRwt patients had undergone a carpal tunnel release procedure, despite carpel tunnel syndrome representing an early symptom in approximately one-third of patients in prior studies [6, 11, 14, 15]. More notable was the lower than expected proportions of ATTR-CM patients who were also diagnosed with heart failure or who were male. ATTR-CM is strongly associated with heart failure, but less than 50\% of the ATTRwt patients also had heart failure. Similarly, ATTRwt has been typically understood to occur predominately in males [11, 15-17], but over $50 \%$ of the patients were female in this study. When only ATTRwt patients diagnosed with heart failure were considered, the proportion of females remained relatively high $(44.8 \%)$.

ATTRwt is likely underdiagnosed, particularly in patients with heart failure with preserved ejection fraction, with one study reporting an ATTRwt incidence of 13\% in patients with heart failure with preserved ejection fraction [18]. The MDV database does not record cases of heart failure with preserved ejection fraction as a unique diagnosis code, coding these patients as heart failure, and it does not record echocardiographic assessments. As a consequence, underdiagnosed or misdiagnosed heart failure with preserved ejection fraction patients may not be captured in the database, which may have contributed to the lower than expected incidence of heart failure in patients with ATTR-CM this study. It has also been shown that a preserved ejection fraction is more common in female than in male heart failure patients [19-21], which may contribute to the higher than expected proportion of female patients identified.

It was notable that the majority of patients in the MDV database did not have a record of a tissue biopsy, suggesting that the diagnosis was made on the basis of clinical criteria. Clinical signs associated with ATTRwt can include evidence of heart failure, increased left ventricular wall thickness, reduced myocardial strain, and a history of carpel tunnel syndrome, but diagnosis is confirmed by demonstrating the presence of transthyretin amyloid in the heart [22, 23]. The number of tissue biopsies may be underestimated as tests conducted at other sites (which are not a part of the database) are not included in the record, although it is not known if this scenario is common. While digestive tract biopsies were much more common than endomyocardial biopsy or abdominal wall 
Table 6 Demographic characteristics of ATTR-CM and subtypes of cardiac amyloidosis patients diagnosed with heart failure (whole period)

\begin{tabular}{|c|c|c|c|c|c|}
\hline & $\begin{array}{l}\text { ATTRwt broad } \\
\text { definition } \\
(N=1798)\end{array}$ & $\begin{array}{l}\text { ATTRwt narrow } \\
\text { definition } \\
(N=1468)\end{array}$ & $\begin{array}{l}\text { ATTRm broad } \\
\text { definition } \\
(N=61)\end{array}$ & $\begin{array}{l}\text { ATTRm narrow } \\
\text { definition } \\
(N=50)\end{array}$ & $\begin{array}{l}\mathrm{AL} \\
\text { amyloidosis } \\
(N=308)\end{array}$ \\
\hline Age (years), mean (SD) & $72.0(12.8)$ & $73.2(11.9)$ & $67.2(13.0)$ & $68.2(12.4)$ & $68.7(11.4)$ \\
\hline Male, $n(\%)$ & $992(55.2)$ & $867(59.1)$ & $36(59.0)$ & $31(62.0)$ & $183(59.4)$ \\
\hline Female, $n(\%)$ & $806(44.8)$ & $601(40.9)$ & $25(41.0)$ & $19(38.0)$ & $125(40.6)$ \\
\hline \multicolumn{6}{|l|}{ Comorbidities $^{\mathrm{a}}, n(\%)$} \\
\hline Heart failure & $1798(100.0)$ & $1468(100.0)$ & $61(100.0)$ & $50(100.0)$ & $308(100.0)$ \\
\hline Arrhythmia & $425(23.6)$ & $351(23.9)$ & $13(21.3)$ & $12(24.0)$ & $59(19.2)$ \\
\hline $\begin{array}{l}\text { Atrial fibrillation or } \\
\text { flutter }\end{array}$ & $596(33.1)$ & $541(36.9)$ & $14(23.0)$ & $12(24.0)$ & $62(20.1)$ \\
\hline Heart block & $172(9.6)$ & $155(10.6)$ & $12(19.7)$ & $9(18.0)$ & $15(4.9)$ \\
\hline Hypertension & $1390(77.3)$ & $1137(77.5)$ & $37(60.7)$ & $30(60.0)$ & $218(70.8)$ \\
\hline $\begin{array}{l}\text { Chronic kidney disease } \\
\text { III-V }\end{array}$ & $718(39.9)$ & $593(40.4)$ & $11(18.0)$ & $8(16.0)$ & $130(42.2)$ \\
\hline Angina & $671(37.3)$ & $564(38.4)$ & $14(23.0)$ & $13(26.0)$ & $110(35.7)$ \\
\hline Diabetes mellitus & $932(51.8)$ & $735(50.1)$ & $25(41.0)$ & $21(42.0)$ & $150(48.7)$ \\
\hline Malignancy & $411(22.9)$ & $326(22.2)$ & $9(14.8)$ & $7(14.0)$ & $256(83.1)$ \\
\hline \multicolumn{6}{|l|}{ Procedures, $n$ (\%) } \\
\hline Carpal tunnel release & $33(1.8)$ & $24(1.6)$ & $1(1.6)$ & $0(0.0)$ & $4(1.3)$ \\
\hline $\begin{array}{l}\text { Surgical aortic valve } \\
\text { replacement }\end{array}$ & $17(0.9)$ & $17(1.2)$ & $0(0.0)$ & $0(0.0)$ & $0(0.0)$ \\
\hline $\begin{array}{l}\text { Transcatheter aortic } \\
\text { valve implantation }\end{array}$ & $15(0.8)$ & $15(1.0)$ & $0(0.0)$ & $0(0.0)$ & $0(0.0)$ \\
\hline \multicolumn{6}{|c|}{ Heart failure medication use, $n(\%)$} \\
\hline Beta-blocker & $795(44.2)$ & $701(47.8)$ & $20(32.8)$ & $16(32.0)$ & $108(35.1)$ \\
\hline ACE inhibitors & $347(19.3)$ & $321(21.9)$ & $11(18.0)$ & $10(20.0)$ & $45(14.6)$ \\
\hline ARBs & $654(36.4)$ & $545(37.1)$ & $14(23.0)$ & $10(20.0)$ & $108(35.1)$ \\
\hline Calcium channel blocker & $842(46.8)$ & $684(46.6)$ & $11(18.0)$ & $7(14.0)$ & $107(34.7)$ \\
\hline Diuretic & $1127(62.7)$ & $984(67.0)$ & $37(60.7)$ & $32(64.0)$ & $243(78.9)$ \\
\hline Aldosterone antagonists & $624(34.7)$ & $577(39.3)$ & $19(31.1)$ & $16(32.0)$ & $124(40.3)$ \\
\hline$\geq 1$ inpatient visit, $n(\%)$ & $1480(82.3)$ & $1237(84.3)$ & $47(77.0)$ & $38(76.0)$ & $274(89.0)$ \\
\hline Death at discharge, $n(\%)$ & $379(21.1)$ & $320(21.8)$ & $6(9.8)$ & $5(10.0)$ & $74(24.0)$ \\
\hline
\end{tabular}


Table 6 continued

\begin{tabular}{llllll}
\hline & $\begin{array}{l}\text { ATTRwt broad } \\
\text { definition } \\
(\boldsymbol{N}=\mathbf{1 7 9 8})\end{array}$ & $\begin{array}{l}\text { ATTRwt narrow } \\
\text { definition } \\
(\boldsymbol{N}=\mathbf{1 4 6 8})\end{array}$ & $\begin{array}{l}\text { ATTRm broad } \\
\text { definition } \\
(\boldsymbol{N}=\mathbf{6 1})\end{array}$ & $\begin{array}{l}\text { ATTRm narrow } \\
\text { definition } \\
(\boldsymbol{N}=\mathbf{5 0})\end{array}$ & $\begin{array}{l}\text { AL } \\
\text { amyloidosis } \\
(\boldsymbol{N}=\mathbf{3 0 8})\end{array}$ \\
\hline $\begin{array}{l}\text { Hospital size, } n(\%) \\
\leq 199 \text { beds }\end{array} \quad 141(7.8)$ & $110(7.5)$ & $3(4.9)$ & $1(2.0)$ & $7(2.3)$ \\
$200-499$ beds & $835(46.4)$ & $724(49.3)$ & $24(39.3)$ & $19(38.0)$ & $110(35.7)$ \\
$\geq 500$ beds & $822(45.7)$ & $634(43.2)$ & $34(55.7)$ & $30(60.0)$ & $191(62.0)$ \\
Hospital administration, $n(\%)$ & & & & \\
University & $270(15.0)$ & $175(11.9)$ & $12(19.7)$ & $11(22.0)$ & $29(9.4)$ \\
$\begin{array}{l}\text { Public (local) } \\
\text { Private }\end{array} 588(32.7)$ & $523(35.6)$ & $27(44.3)$ & $25(50.0)$ & $88(28.6)$ \\
$\begin{array}{l}\text { Public } \\
\text { (government) }\end{array}$ & $583(19.6)$ & $276(18.8)$ & $7(11.5)$ & $3(6.0)$ & $55(17.9)$ \\
\hline
\end{tabular}

From Jan 2010 to Sep 2018

$A C E$ angiotensin-converting enzyme, $A R B$ angiotensin receptor blocker, ATTRwt wild-type transthyretin amyloid cardiomyopathy, ATTRm hereditary transthyretin amyloidosis, $S D$ standard deviation

a Comorbidities occurring in $>15 \%$ of ATTR-CM patients (broad definition)

biopsy, endomyocardial biopsy would typically be necessary for a formal histological diagnosis [23]. Very few patients had a digestive tract biopsy and an endomyocardial biopsy, particularly within 8 weeks of diagnosis, suggesting that the majority of the digestive tract biopsies may have been an artefact unrelated to the diagnosis of ATTR-CM. This was not unexpected, as digestive tract biopsy is a common test in Japan. Nevertheless, the fact that over 400 ATTRwt patients had a digestive tract biopsy within 8 weeks of diagnosis could be a significant finding as it implies that it may be an underestimated diagnostic modality in Japan. However, since only a small proportion $(\sim 9 \%)$ of the ATTRwt patients had an endomyocardial biopsy or abdominal wall biopsy at some point (and even fewer within 8 weeks of diagnosis $[\sim 4 \%])$, there is the possibility that this study overestimates the prevalence. The immunostaining of tissue biopsy to identify transthyretin can be associated with a false positive rate, depending on the methodology used $[7,14]$, so this may also contribute to an overestimation. At the same time, this study does not capture patients who were misdiagnosed or were undiagnosed due to less severe disease, and may therefore underestimate the prevalence.

As a retrospective study of data from a secondary source, this analysis may be limited by errors, omissions, or variations in the source records. This was not a longitudinal study, patients were not followed over time, and patient outcomes and mortality rates could not be assessed. This limitation means that patients' prognoses and outcomes could not be assessed. The MDV database is limited to hospitals with $\geq 20$ beds and, as such, does not include smaller clinics.

\section{CONCLUSION}

This retrospective study revealed that in the MDV database in Japan over the 9 years of this analysis, between 3255 and 3992 patients were diagnosed with ATTRwt, and between 67 and 106 patients were diagnosed with ATTRm. Limited to patients who were also diagnosed with heart failure, there were between 1468 and 1798 diagnoses of 


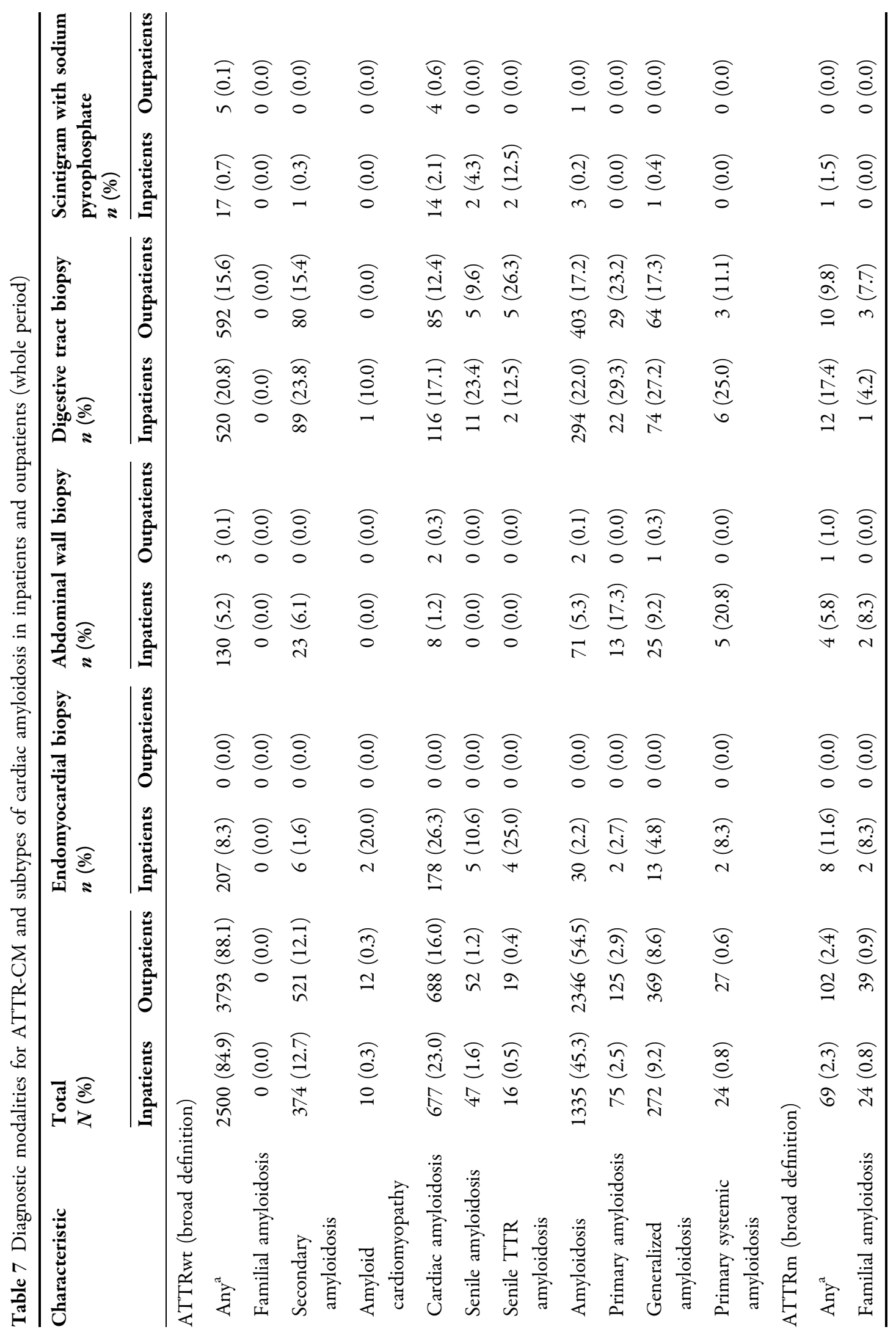




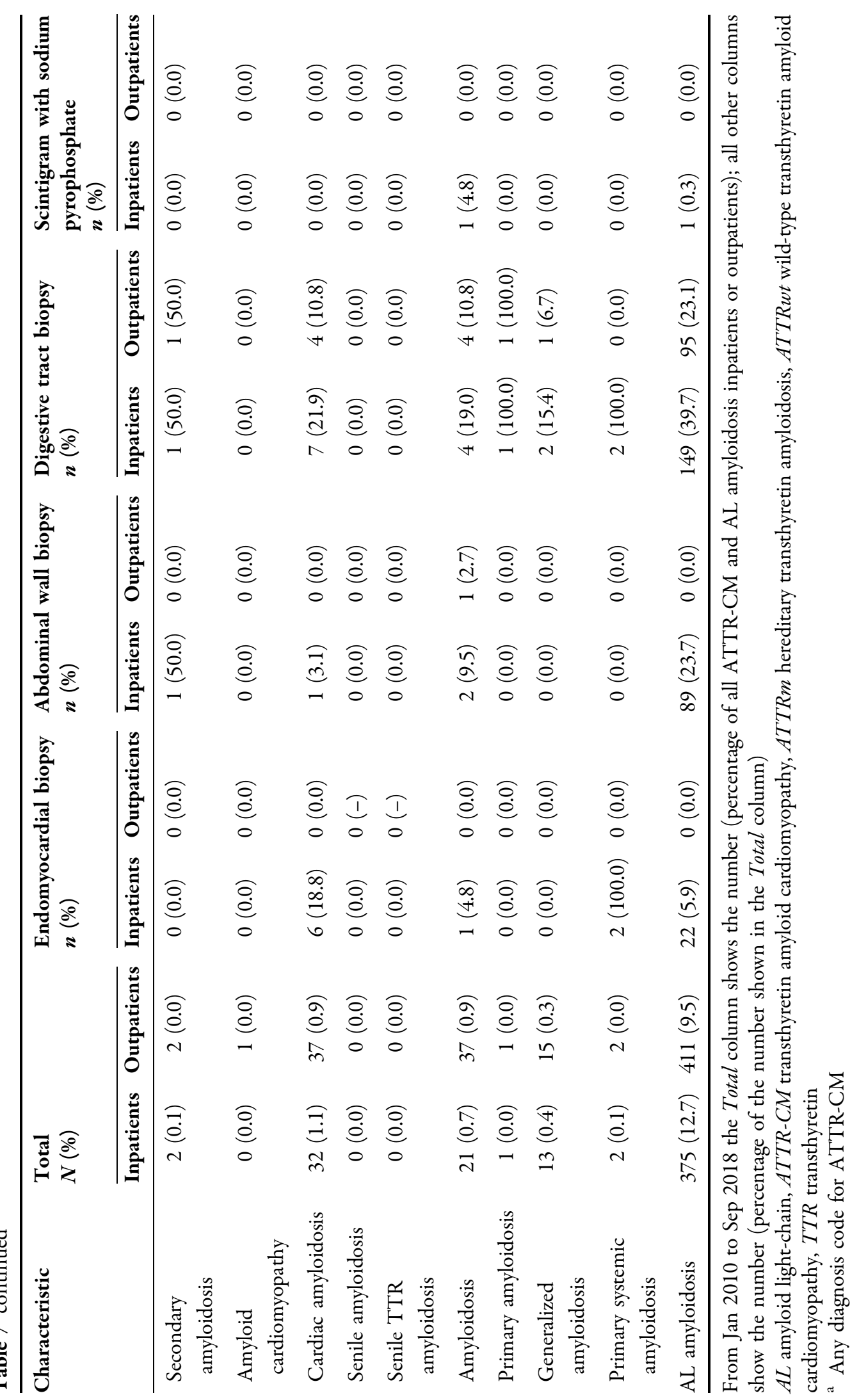


Table 8 Diagnostic modalities for ATTR-CM and subtypes of cardiac amyloidosis patients diagnosed with heart failure (whole period)

\begin{tabular}{|c|c|c|c|c|c|}
\hline & $\begin{array}{l}\text { Total } \\
N(\%)\end{array}$ & $\begin{array}{l}\text { Endomyocardial } \\
\text { biopsy } \\
n(\%)\end{array}$ & $\begin{array}{l}\text { Abdominal } \\
\text { wall biopsy } \\
n(\%)\end{array}$ & $\begin{array}{l}\text { Digestive tract } \\
\text { biopsy } \\
n(\%)\end{array}$ & $\begin{array}{l}\text { Scintigram } \\
\text { with sodium } \\
\text { pyrophosphate } \\
n(\%)\end{array}$ \\
\hline \multicolumn{6}{|l|}{ ATTRwt (broad definition) } \\
\hline Any $^{a}$ & $1798(83.0)$ & $197(11.0)$ & $63(3.5)$ & $494(27.5)$ & $21(1.2)$ \\
\hline Familial amyloidosis & $0(0.0)$ & $0(0.0)$ & $0(0.0)$ & $0(0.0)$ & $0(0.0)$ \\
\hline Secondary amyloidosis & $221(10.2)$ & $5(2.3)$ & $15(6.8)$ & $68(30.8)$ & $1(0.5)$ \\
\hline Amyloid cardiomyopathy & $10(0.5)$ & $2(20.0)$ & $0(0.0)$ & $1(10.0)$ & $0(0.0)$ \\
\hline Cardiac amyloidosis & $686(31.7)$ & $170(24.8)$ & $8(1.2)$ & $170(24.8)$ & $17(2.5)$ \\
\hline Senile amyloidosis & $37(1.7)$ & $5(13.5)$ & $0(0.0)$ & $12(32.4)$ & $2(5.4)$ \\
\hline Senile TTR amyloidosis & $16(0.7)$ & $4(25.0)$ & $0(0.0)$ & $4(25.0)$ & $2(12.5)$ \\
\hline Amyloidosis & $799(36.9)$ & $27(3.4)$ & $36(4.5)$ & $251(31.4)$ & $4(0.5)$ \\
\hline Primary amyloidosis & $48(2.2)$ & $2(4.2)$ & $1(2.1)$ & $16(33.3)$ & $0(0.0)$ \\
\hline Generalized amyloidosis & $239(11.0)$ & $13(5.4)$ & $15(6.3)$ & $73(30.5)$ & $1(0.4)$ \\
\hline Primary systemic amyloidosis & $16(0.7)$ & $1(6.3)$ & $1(6.3)$ & $3(18.8)$ & $0(0.0)$ \\
\hline \multicolumn{6}{|l|}{ ATTRm (broad definition) } \\
\hline Any $^{a}$ & $61(2.8)$ & $8(13.1)$ & $2(3.3)$ & $17(27.9)$ & $1(1.6)$ \\
\hline Familial amyloidosis & $18(0.8)$ & $2(11.1)$ & $0(0.0)$ & $2(11.1)$ & $0(0.0)$ \\
\hline Secondary amyloidosis & $2(0.1)$ & $0(0.0)$ & $1(50.0)$ & $2(100.0)$ & $0(0.0)$ \\
\hline Amyloid cardiomyopathy & $1(0.0)$ & $0(0.0)$ & $0(0.0)$ & $0(0.0)$ & $0(0.0)$ \\
\hline Cardiac amyloidosis & $34(1.6)$ & $6(17.6)$ & $1(2.9)$ & $11(32.4)$ & $0(0.0)$ \\
\hline Senile amyloidosis & $0(0.0)$ & $0(0.0)$ & $0(0.0)$ & $0(0.0)$ & $0(0.0)$ \\
\hline Senile TTR amyloidosis & $0(0.0)$ & $0(0.0)$ & $0(0.0)$ & $0(0.0)$ & $0(0.0)$ \\
\hline Amyloidosis & $23(1.1)$ & $1(4.3)$ & $2(8.7)$ & $6(26.1)$ & $1(4.3)$ \\
\hline Primary amyloidosis & $1(0.0)$ & $0(0.0)$ & $0(0.0)$ & $1(100.0)$ & $0(0.0)$ \\
\hline Generalized amyloidosis & $10(0.5)$ & $0(0.0)$ & $0(0.0)$ & $2(20.0)$ & $0(0.0)$ \\
\hline Primary systemic amyloidosis & $2(0.1)$ & $2(100.0)$ & $0(0.0)$ & $2(100.0)$ & $0(0.0)$ \\
\hline AL amyloidosis & $308(14.2)$ & $22(7.1)$ & $58(18.8)$ & $159(51.6)$ & $1(0.3)$ \\
\hline
\end{tabular}

From Jan 2010 to Sep 2018 the Total column shows the number (percentage of all ATTR-CM and AL amyloidosis inpatients or outpatients); all other columns show the number (percentage of the number shown in the Total column) $A L$ amyloid light-chain, ATTR-CM transthyretin amyloid cardiomyopathy, ATTRm hereditary transthyretin amyloidosis, $A T T R w t$ wild-type transthyretin amyloid cardiomyopathy, TTR transthyretin

a Any diagnosis code for ATTR-CM 
ATTRwt and between 50 and 61 diagnoses of ATTRm. As awareness of the disease grows, it is likely that more patients will be diagnosed, and further studies on the true prevalence of ATTR$\mathrm{CM}$ should be undertaken in the future. Greater awareness of the disease, together with the emergence of new treatments, may also improve diagnosis and treatment outcomes.

\section{ACKNOWLEDGEMENTS}

We thank Dr. Jin Endo for helpful discussions on this study.

Funding. This study was sponsored by Pfizer, which also funded the journal's Rapid Service Fee. All authors had full access to all of the data in this study and take complete responsibility for the integrity of the data and accuracy of the data analysis.

Medical Writing Assistance. Medical writing support was provided by Joshua Fink, PhD, of Engage Scientific Solutions, and was funded by Pfizer.

Authorship. All named authors meet the International Committee of Medical Journal Editors (ICMJE) criteria for authorship for this article, take responsibility for the integrity of the work as a whole, and have given their approval for this version to be published.

Disclosures. Ian Winburn is a full-time employee of Pfizer and holds stock and/or stock options with Pfizer. Tomonori Ishii is a full-time employee of Pfizer and holds stock and/or stock options with Pfizer. Takuma Sumikawa is a fulltime employee of Pfizer and holds stock and/or stock options with Pfizer. Kanae Togo is a fulltime employee of Pfizer and holds stock and/or stock options with Pfizer. Hideo Yasunaga has nothing to disclose.

Compliance with Ethics Guidelines. This article is based on previously conducted studies and does not contain any studies with human participants or animals performed by any of the authors. All patient data are anonymized with no personal data, so informed consent was not required.

Data Availability. Pfizer provides secure access to anonymized patient-level data to qualified researchers in response to scientifically valid research proposals. Further details can be found at: http://www.pfizer.com/research/ clinical_trials/trial_data_and_results/data_ requests.

Open Access. This article is distributed under the terms of the Creative Commons Attribution-NonCommercial 4.0 International License (http://creativecommons.org/licenses/ by-nc/4.0/), which permits any noncommercial use, distribution, and reproduction in any medium, provided you give appropriate credit to the original author(s) and the source, provide a link to the Creative Commons license, and indicate if changes were made.

\section{REFERENCES}

1. Ruberg FL, Berk JL. Transthyretin (TTR) cardiac amyloidosis. Circulation. 2012;126:1286-300.

2. Izumiya $Y$, Takashio $S$, Oda S, et al. Recent advances in diagnosis and treatment of cardiac amyloidosis. J Cardiol. 2018;71:135-43.

3. Siddiqi OK, Ruberg FL. Cardiac amyloidosis: an update on pathophysiology, diagnosis, and treatment. Trends Cardiovasc Med. 2018;28:10-21.

4. Castano A, Narotsky DL, Hamid N, et al. Unveiling transthyretin cardiac amyloidosis and its predictors among elderly patients with severe aortic stenosis undergoing transcatheter aortic valve replacement. Eur Heart J. 2017;38:2879-87.

5. Konishi M, Ishida J, Springer J, et al. Heart failure epidemiology and novel treatments in Japan: facts and numbers. ESC Heart Fail. 2016;3:145-51.

6. Sekijima Y, Uchiyama S, Tojo K, et al. High prevalence of wild-type transthyretin deposition in patients with idiopathic carpal tunnel syndrome: a common cause of carpal tunnel syndrome in the elderly. Hum Pathol. 2011;42:1785-91.

7. Sekijima Y, Yazaki M, Ueda M, et al. First nationwide survey on systemic wild-type ATTR amyloidosis in Japan. Amyloid. 2018;25:8-10. 
8. Isogai $\mathrm{T}$, Yasunaga $\mathrm{H}$, Matsui $\mathrm{H}$, et al. Hospital volume and cardiac complications of endomyocardial biopsy: a retrospective cohort study of 9508 adult patients using a nationwide inpatient database in Japan. Clin Cardiol. 2015;38:164-70.

9. Statistics Bureau, Ministry of Internal Affairs and Communications Japan (2018) Monthly report. http://www.stat.go.jp/english/data/jinsui/tsuki/ index.html. Accessed 13 Mar 2019

10. Damy T, Costes B, Hagege AA, et al. Prevalence and clinical phenotype of hereditary transthyretin amyloid cardiomyopathy in patients with increased left ventricular wall thickness. Eur Heart J. 2016;37:1826-34.

11. Maurer MS, Hanna M, Grogan M, et al. Genotype and Phenotype of Transthyretin Cardiac Amyloidosis: THAOS (Transthyretin Amyloid Outcome Survey). J Am Coll Cardiol. 2016;68:161-72.

12. Damy T, Kristen AV, Suhr OB, et al. Transthyretin cardiac amyloidosis in continental Western Europe: an insight through the Transthyretin Amyloidosis Outcomes Survey (THAOS). Eur Heart J. https://doi. org/10.1093/eurheartj/ehz173

13. Lane T, Fontana M, Martinez-Naharro A, et al. Natural history, quality of life, and outcome in cardiac transthyretin amyloidosis. Circulation 2019;140:16-26.

14. Gertz MA, Benson MD, Dyck PJ, et al. Diagnosis, prognosis, and therapy of transthyretin amyloidosis. J Am Coll Cardiol. 2015;66:2451-66.

15. Gonzalez-Lopez E, Gagliardi C, Dominguez F, et al. Clinical characteristics of wild-type transthyretin cardiac amyloidosis: disproving myths. Eur Heart J. 2017;38:1895-904.

16. Connors LH, Sam F, Skinner M, et al. Heart failure resulting from age-related cardiac amyloid disease associated with wild-type transthyretin: a prospective, observational cohort study. Circulation. 2016;133:282-90.

17. Pinney JH, Whelan CJ, Petrie A, et al. Senile systemic amyloidosis: clinical features at presentation and outcome. J Am Heart Assoc. 2013;2:e000098.

18. Gonzalez-Lopez E, Gallego-Delgado M, GuzzoMerello G, et al. Wild-type transthyretin amyloidosis as a cause of heart failure with preserved ejection fraction. Eur Heart J. 2015;36:2585-94.

19. Tsuchihashi-Makaya M, Hamaguchi S, Kinugawa S, et al. Characteristics and outcomes of hospitalized patients with heart failure and reduced vs preserved ejection fraction. Report from the Japanese Cardiac Registry of Heart Failure in Cardiology (JCARECARD). Circ J. 2009;73:1893-900.

20. Owan TE, Hodge DO, Herges RM, et al. Trends in prevalence and outcome of heart failure with preserved ejection fraction. $\mathrm{N}$ Engl $\mathrm{J}$ Med. 2006;355:251-9.

21. Vedin O, Lam CSP, Koh AS, et al. Significance of ischemic heart disease in patients with heart failure and preserved, midrange, and reduced ejection fraction: a Nationwide Cohort Study. Circ Heart Fail. 2017;10:e003875.

22. Elliott PM, Anastasakis A, Borger MA, et al. 2014 ESC guidelines on diagnosis and management of hypertrophic cardiomyopathy: the Task Force for the Diagnosis and Management of Hypertrophic Cardiomyopathy of the European Society of Cardiology (ESC). Eur Heart J. 2014;35:2733-79.

23. Rapezzi C, Quarta CC, Riva L, et al. Transthyretinrelated amyloidoses and the heart: a clinical overview. Nat Rev Cardiol. 2010;7:398-408. 